Pacific Journal of Mathematics

MRESENTITIONS BY SPROG GEN 


\title{
REPRESENTATIONS BY SPINOR GENERA
}

\author{
J. S. HSIA
}

If $f$ and $g$ are two nonsingular quadratic forms with rational integral coefficients such that $f$ represents $g$ integrally over every $p$-adic fields and also over the reals, then it is a wellknown classical result that the genus Gen $(f)$ of $f$ represents $g$. This paper considers the question of how many spinor genera in the genus of $f$ will represent $g$, when $f$ and $g$ are integral forms defined over some fixed domain of algebraic integers and when $\operatorname{dim}(f)-\operatorname{dim}(g) \geqq 2$.

Unless otherwise mentioned the following general assumptions will be understood throughout this paper: $F$ is an algebraic number field with $R$ as its ring of algebraic integers, $V$ and $W$ are finite dimensional regular quadratic spaces over $F$ with $\operatorname{dim} V-\operatorname{dim} W=$ $d \geqq 2, L$ and $K$ are respectively $R$-lattices on $V$ and $W$, and $S$ is the set of all discrete spots on $F$. All unexplained notations and terminologies are from [6]. Suppose now that $L_{\mathfrak{p}}$ represents $K_{\mathfrak{p}}$ for every $\mathfrak{p} \in S$, then it is a well-known result that there is a lattice $L^{\prime}$ in the genus of $L$ that represents $K$, provided $V$ represents $W$ (in fact, if $W$ were a subspace of $V$, this $L^{\prime}$ may be chosen so as to contain $K$; see 102:5, [6]). We introduce the notations $K \rightarrow$ Gen $(L), \operatorname{Spn}(L), \mathrm{Spn}^{+}(L), \mathrm{Cls}(L), \mathrm{Cls}^{+}(L)$ to denote respectively that $K$ is representable by a member in the genus, spinor genus, proper spinor genus, class, proper class of $L$. Thus, in this notation, $L_{\text {}}$ represents $K_{\mathfrak{p}}$ locally everywhere at $\mathfrak{p} \in S$ and $W \rightarrow V$ is equivalent to $K \rightarrow \operatorname{Gen}(L)$, which is, of course, the same as representation by $\operatorname{Gen}^{+}(L)$. We show here that if $d \geqq 3$ then $K \rightarrow$ Gen $(L)$ implies $K \rightarrow \mathrm{Spn}^{+}(L)$ so that in the indefinite case for $L$ every proper class in the genus represents $K$. This fact must surely have been known to the specialists although $I$ have not seen it in print and choose to record it here for completeness; its proof is quite standard and does not employ any of the subtler or deeper aspects of the theory. On the other hand, when $d=2$, the theory is a good deal more intricate. We show that here too in most cases $K$ is representable by every proper spinor genus in the genus of $L$; the exceptional cases will be pointed out, and there one needs to know the precise results for the local computations of the spinor norms of local integral rotations on $L_{p}$; the known facts about these are found in [3] for nondyadic $\mathfrak{p}$, in [1] for unramified dyadic $\mathfrak{p}$, and in [2] for arbitrary dyadic $\mathfrak{p}$ but with $L_{p}$ modular. This study was motivated by Kneser's paper [4], and the results as well as the method follow closely along his 
lines with some refinements necessitated by handling here also the cases where $L$ may not be totally indefinite. Indeed, it may be definite.

Suppose for all spots on $F$ we are given that $K_{p} \rightarrow L_{p}$, then surely $F_{\mathfrak{p}} K_{\mathfrak{p}} \rightarrow F_{\mathfrak{p}} L_{\mathfrak{p}}$ for every $\mathfrak{p}$. Hence, Hasse-Minkowski implies $W \rightarrow V$. Thus, we may as well assume at the outset that $W$ is a regular subspace of $V$, and $K$ is a lattice in $V$. Write $V=W \perp U$, and $\delta$ the discriminant (in O'Meara's sense) of $U$. As in Example 102: 5, [6] if we let $T=\left\{\mathfrak{p} \in S: L_{\mathfrak{p}} \nsupseteq K_{\mathrm{p}}\right\}$, then $T$ is a finite set. By lattice theory, there is a lattice $L^{\prime}$ on $V$ such that

$$
L_{\mathfrak{p}}^{\prime}= \begin{cases}L_{\mathfrak{p}} & \text { for } \quad \mathfrak{p} \notin T \\ s_{\mathfrak{p}} L_{\mathfrak{p}} & \text { for } \quad \mathfrak{p} \in T .\end{cases}
$$

Here $s_{\mathfrak{p}} \in O\left(V_{\mathfrak{p}}\right)$, and the hypotheses permits us to choose $s_{\mathfrak{p}}$ so that $s_{p} L_{p} \supseteqq K_{p}$. Clearly, $s_{p}$ can be assumed to be in $O^{+}\left(V_{p}\right)$. Should $d \geqq 3$, then $\theta\left(O^{+}\left(U_{p}\right)\right)=F_{p}^{\times}$by 91: 6, [6]. Therefore, we may find a rotation

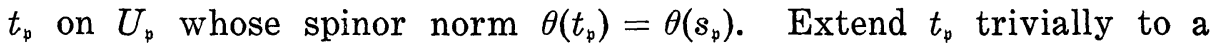

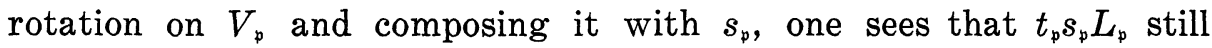
contains $K_{\mathfrak{p}}$. Thus, we may further assume that our original $s_{\mathfrak{p}}$ belongs to $O^{\prime}\left(V_{\mathfrak{p}}\right)$. This shows that $L^{\prime}$ belongs to the proper spinor genus of $L$. Thus, we have: if $L_{\mathfrak{p}}$ represents $K_{\mathfrak{p}}$ at every (finite and infinite) spot $\mathfrak{p}$ and if $d=r k(L)-r k(K) \geqq 3$, then $K$ is represented by every proper spinor genus in the genus of $L$; in particular, if $L$ is indefinite with respect to $S$, then $K$ is representable by every proper class in Gen $(L)$.

REMARK. Specializing this statement to the case when $L$ is indefinite, $r k(K)=1, F=\boldsymbol{Q}$, a theorem of Watson's [9] is recaptured. Suppose we permit $W$ to be a degenerate space, say the radical $\operatorname{Rad}(W)$ has dimension $r$. Then, the same result prevails provided we have: $r k(L)-r k(K)=d \geqq 3+r$. To see this, note that one can embed $W$ in a nonsingular space $\widetilde{W}=\boldsymbol{H} \perp W_{a n}$, where $W_{a n}$ is the anisotropic kernel of $W$, and $\boldsymbol{H}$ a hyperbolic space of dimension $2 r$.

From here onward we assume that $d=2$. Clearly, the group $J_{U}$ of split rotations (adèles) on $U$ may be viewed as a subgroup of $J_{V}$; similarly $P_{V}, J_{V}^{\prime}, J_{L}$ are defined in Chapter $\mathrm{X}$, [6], as are the following subgroups of the idèle group $J_{F}$ of $F: P_{F}, P_{D}, J_{F}^{L}$. We ask the following two basic questions:

(A): If $L$ represents $K$ properly, is it true that for every $\phi \in J_{U}$ we have $K \rightarrow \operatorname{Spn}^{+}(\phi(L))$ ?

(B): What is the group index $\left[J_{V}: J_{U} P_{V} J_{V}^{\prime} J_{L}\right]$ ? 
We shall see below that Question (A) has an affirmative answer (Theorem 1); and Theorem 2 will show that this group index mentioned above is less or equal to two. This means that at least half (if not all) of the proper spinor genera in the genus of $L$ will represent $K$, provided there is just one lattice in the genus that represents $K$.

To treat Question (A), suppose $s: K \rightarrow L$ is a proper representation of $K$ by $L$, choose any full lattice $N$ on $U$ (recall that $V=W \perp U$ ), and set $K^{\prime}=K \perp N$. If $\phi \in J_{U}$ is given, clearly, the lattice $\phi\left(K^{\prime}\right)$ is just the lattice $K \perp \phi(N)$. If we select our $N$ above to be the lattice $s^{-1}(L) \cap U$, then $s\left(K^{\prime}\right)=s(K \perp N)=s(K) \perp s\left(s^{-1}(L) \cap U\right) \subseteq L+L=L$. Therefore, $s$ also induces a proper representation of $K^{\prime}$ by $L$. Now, $r k\left(K^{\prime}\right)=r k(L)$. We have $s^{-1}(L) \supseteqq K^{\prime}$ so that $\phi s^{-1}(L) \supseteqq \phi\left(K^{\prime}\right)$. Since $J_{V}^{\prime}$ contains the commutator subgroup of $J_{V}, J_{V} / P_{V} J_{V}^{\prime}$ is abelian. Hence, $\phi s^{-1} \in \phi P_{V} \subseteq \phi P_{V} J_{V}^{\prime}=P_{V} J_{V}^{\prime} \phi$. This means there is a lattice in the proper spinor genus of $\phi(L)$ that represents $\phi\left(K^{\prime}\right)$. But, $\phi$ is trivial on $K$ and therefore, we obtain:

THEOREM 1. If $K \rightarrow \mathrm{Cls}^{+}(L)$, and $d=r k(L)-r k(K)=2$, then $K \rightarrow \operatorname{Spn}^{+}(\phi(L))$ for every $\phi \in J_{U}$, where $F L=F K \perp U$. In particular, if $L$ is indefinite with respect to the defining set $S$ of spots on $F$, then $\phi(L)$ represents $K$ properly for all such $\phi$.

Put $E=F(\sqrt{-\delta})$, where $\delta=\operatorname{disc}(U)$, and $D=\theta\left(O^{+}(V)\right)$. The map from $J_{V}$ into $J_{F}$ given by $s=\left(s_{\mathfrak{p}}\right) \mapsto j=\left(j_{\mathfrak{p}}\right)$ where $j_{\mathfrak{p}} \in \theta\left(s_{\mathfrak{p}}\right)$ induces a monomorphism $\phi_{L}: J_{V} / P_{V} J_{V}^{\prime} J_{L} \nrightarrow J_{F} / P_{D} J_{F}^{L}$ (which is an isomorphism when $r k(L) \geqq 3)$. As a preliminary step toward determining the group index in Question (B), we have:

LEMMA. $\phi_{L}$ described above induces an isomorphism:

$$
\phi_{L, K}: J_{V} / J_{U} P_{V} J_{V}^{\prime} J_{L} \longrightarrow J_{F} / P_{D} N_{E / F}\left(J_{E}\right) J_{F}^{L} \text {. }
$$

Proof. If $-\delta$ is a square in $F$, then $E=F$, and the right hand side in $(*)$ is trivial. But then, $U$ is a hyperbolic plane and $\theta\left(O^{+}\left(U_{p}\right)\right)=$ $F_{b}^{\times}$. Hence, every split rotation on $V$ may be composed with one $U$ so as to have the resulting element lying inside $J_{V}^{\prime}$, and this implies the left hand side of $(*)$ also is trivial. Therefore, we may suppose that $-\delta$ is a nonsquare. If $s \in J_{U}$, then $\theta\left(s_{\mathfrak{p}}\right) \in \theta\left(O^{+}\left(U_{\mathfrak{p}}\right)\right)=\theta\left(O^{+}(\langle 1, \delta\rangle)\right)=$ $Q(\langle 1, \delta\rangle) F_{\mathfrak{p}}^{\times 2}=N_{E_{\mathfrak{p}} / F_{\mathfrak{p}}}\left(E_{\mathfrak{p}}^{\times}\right)$for $\mathfrak{P} \mid \mathfrak{p}$. Thus, the map which sends $J_{V}$ into $J_{F}$ mentioned above also sends $J_{U}$ into $N_{E / F}\left(J_{E}\right)$ so that the map $\phi_{L, K}: J_{V} / J_{U} P_{V} J_{V}^{\prime} J_{L} \rightarrow J_{F} / P_{D} N_{E / F}\left(J_{E}\right) J_{F}^{L}$ is well induced by $\phi_{L}$. Since $r k(K)>0, r k(L) \geqq 3$ necessarily so that our $\phi_{L, K}$ must be surjective as well by the above discussion. To see the kernel is precisely 
$J_{U} P_{V} J_{V}^{\prime} J_{L}$, consider $s \in J_{V}$ such that $\phi_{L, K}(\bar{s})=\overline{1}$. This means that $\theta\left(s_{\mathfrak{p}}\right)=a j_{\mathfrak{p}} i_{\mathfrak{p}} F_{\mathfrak{p}}^{\times 2}$, where $a \in D, j=\left(j_{\mathfrak{p}}\right) \in N_{E / F}\left(J_{E}\right)$, and $i=\left(i_{\mathfrak{p}}\right) \in J_{F}^{L}$. Write $a=\theta(f), f \in 0^{+}(V), i_{\mathfrak{p}}=\theta\left(\Sigma_{\mathfrak{p}}\right), \Sigma_{\mathfrak{p}} \in O^{+}\left(L_{\mathfrak{p}}\right)$. For each $\mathfrak{P} \mid \mathfrak{p}$, the local norm $N_{\mathfrak{p} \mid \downarrow}\left(E_{\Re}^{\times}\right)$is either all of $F_{p}^{\times}$or it is a subgroup $Q(\langle 1, \delta\rangle)$ of index two in $F_{\mathfrak{p}}^{\times}$. Therefore, we can find a local rotation $h_{\mathfrak{p}}$ on $U_{p}$ such that $\theta\left(h_{\mathfrak{p}}\right)=j_{\mathfrak{p}} F_{\mathfrak{p}}^{\times 2}$. Thus, $\theta\left(s_{\mathfrak{p}}\right)=\theta(f) \theta\left(h_{\mathfrak{p}}\right) \theta\left(\Sigma_{\mathfrak{p}}\right)$ which implies

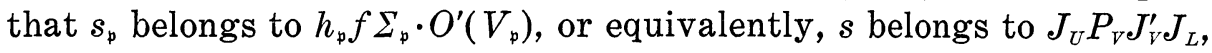
proving the lemma.

This lemma translates the index computation in Question (B) to an equivalent one in terms of idèles, which is usually more manageable, and we now take up this calculation.

LEMma. $\left[J_{F}: P_{D} N_{E / F}\left(J_{E}\right)\right]=2 \cdot\left[F^{\times}: D\right]$.

Proof. Here $D$ is characterized as the set of nonzero field elements from $F$ that are positive at all real spots $\mathfrak{p}$ for which the quadratic space $V_{\uparrow}$ is definite. See 101:8, [6]. Let $R$ denote the set of such real spots on $F$. Note that if $\operatorname{Card}(R)=t$, then $F^{\times} / D$ is a vector space of dimension $t$ over $\boldsymbol{F}_{2}$ It is well-known that $\left[J_{F}: P_{F} N_{E / F}\left(J_{E}\right)\right]=2$; see $65: 21$, [6]. Therefore, $\left[J_{F}: P_{D} N_{E / F}\left(J_{E}\right)\right]=$ $2 \cdot\left[P_{F} N_{E / F}\left(J_{E}\right): P_{D} N_{E / F}\left(J_{E}\right)\right]=2\left[P_{F}: P_{F} \cap P_{D} N_{E / F}\left(J_{E}\right)\right]=2 \cdot\left[P_{F}: P_{D}\right]=$ $2 \cdot\left[F^{\times}: D\right]$. Only the second last equality requires some explanations. If $x \in F^{\times}$, and $d \in D$, then $x / d$ belongs to $N_{E / F}\left(J_{E}\right)$ implies, in particular, that at each real spot $\mathfrak{p}$ from $R, x / d$ is a local norm at $\mathfrak{p}$. But $V_{\mathfrak{p}}$ is anisotropic so that $-\delta$ is a nonsquare at $\mathfrak{p}$. Hence, the local norms at $\mathfrak{p}$ consist of all the positive reals. This means $x / d$ is positive at $\mathfrak{p}$, and so $x$ is positive at $\mathfrak{p}$. Therefore, $x \in D$.

LEMma. $J_{F}^{L} \subseteq P_{D} N_{E / F}\left(J_{E}\right)$ if and only if $J_{F}^{L} \subseteq N_{E / F}\left(J_{E}\right)$.

Proof. This is the type of result that is typically bewildering and yet at the same time powerfully evident of the beauty and depth of the arithmetic of global fields. For the proof, clearly it suffices to prove the "only if" part. $L_{\uparrow}$ is unimodular almost everywhere. So, let $T$ be the set of discrete spots $\mathfrak{p}$ on $F$ for which $\theta\left(O^{+}\left(L_{p}\right)\right)$ is not contained in $\left(N_{E / F}\left(J_{E}\right)\right)_{p}$. So, $T$ is a finite set. If $T$ is not empty, there must be an $x_{\mathfrak{p}} \in \theta\left(O^{+}\left(L_{\mathfrak{p}}\right)\right)$ not lying in $N_{E_{\mathfrak{F}^{\prime}} F_{\mathfrak{p}}}\left(E_{\Re}^{\times}\right)$ for $\mathfrak{P} \mid \mathfrak{p}$. This means $x_{\mathfrak{p}}$ is not represented by the binary quadratic space $\langle 1, \delta\rangle$ over $F_{p}$. Consider the idèle $i=\left(i_{p}\right)$ where $i_{q}=x_{p}$ at $\mathfrak{q}=\mathfrak{p}$, and $i_{\mathfrak{q}}=1$ elsewhere. Surely, $j$ belongs to $J_{F}^{L}$. Hence, by hypotheses, there exists $d \in D$ such that $d j \in N_{E / F}\left(J_{E}\right)$. This means $d$ is a local norm at all the spots $\mathfrak{q} \neq \mathfrak{p}$. Hence, by Hilbert Reciprocity Law, $d$ is also a local norm at $\mathfrak{p}$. On the other hand, $d x_{\mathfrak{p}}$ is a local 
norm at $\mathfrak{p}$. So, we arrive at a contradictory conclusion that $x_{\mathfrak{p}}$ is a local norm after all. Therefore, $T$ must be empty and $J_{F}^{L} \subseteq N_{E / F}\left(J_{E}\right)$.

\section{THEOREM 2. $\left[J_{F}: P_{D} N_{E / F}\left(J_{E}\right) J_{F}^{L}\right] \leqq 2$.}

Proof. If $D=F^{\times}$(i.e. when $L$ is totally indefinite with respect to $S)$, then already $\left[J_{F}: P_{F} N_{E / F}\left(J_{E}\right)\right]=2$ in which case the index is two if and only if $J_{F}^{L}$ is contained in $N_{E / F}\left(J_{E}\right)$ by lemma. So, we may assume that $D \neq F^{\times}$.

Let $R=\left\{\mathfrak{p}_{1}, \cdots \mathfrak{p}_{t}\right\}$ be the set of all real spots on $F$ for which $V_{\mathfrak{p}_{i}}$ is anisotropic, and let $e_{i}(1 \leqq i \leqq t)$ be the idèle which has -1 as its component at $\mathfrak{p}_{i}$ and 1 elsewhere. Clearly, all these $e_{i}$ 's belong to $J_{F}^{L}$ but not to $N_{E / F}\left(J_{E}\right)$, and therefore, also not to $P_{D} N_{E / F}\left(J_{E}\right)$ by Lemma. Define the chain of subgroups of $P_{D} N_{E / F}\left(J_{E}\right) J_{F}^{L}$ by: $G_{0}=$ $P_{D} N_{E / F}\left(J_{E}\right), G_{j}=I_{F}\left(p_{j}\right) G_{j-1}$ for $1 \leqq j \leqq t$, where $I_{F}\left(\mathfrak{p}_{j}\right)$ denotes the group of idèles which have all the components different from $\mathfrak{p}_{j}$ the value 1. Thus, we have an increasing tower:

$$
P_{D} N_{E / F}\left(J_{E}\right)=G_{0} \leqq \cdots \leqq G_{t} .
$$

We assert that all the inclusions are strict. If not, we shall have at some $j, I_{F}\left(\mathfrak{p}_{j}\right) \subseteq G_{j-1}=I_{F}\left(\mathfrak{p}_{1}\right) \cdots I_{F}\left(\mathfrak{p}_{j-1}\right) P_{D} N_{E / F}\left(J_{E}\right)$. The idèle $e_{j}$ surely belongs to $I_{F}\left(\mathfrak{p}_{j}\right)$ and this means for some $d \in D$ and $\eta \in$ $I_{F}\left(\mathfrak{p}_{1}\right) \cdots I_{F}\left(\mathfrak{p}_{j-1}\right) N_{E / F}\left(J_{E}\right)$ one has $e_{j}=(d) \eta$. But, at all the spots outside of $R, \eta_{p}=d^{-1}$ which implies that $d^{-1}$, hence also $d$, is a local norm. Inside of $R$ the element $d$ is positive at each $\mathfrak{p}$ and so is also a local norm. Thus, $d$ is itself a global norm. Therefore, we conclude that $e_{j}$ belongs to $I_{F}\left(\mathfrak{p}_{1}\right) \cdots I_{F}\left(\mathfrak{p}_{j-1}\right) N_{E / F}\left(J_{E}\right)$. On the other hand, at $\mathfrak{p}_{j}, e_{j}$ is negative whereas every element from $I_{F}\left(\mathfrak{p}_{1}\right) \cdots I_{F}\left(\mathfrak{p}_{j-1}\right) N_{E / F}\left(J_{E}\right)$ has positive component at $\mathfrak{p}_{j}$. This contradiction proves our assertion.

Since for each $j(1 \leqq j \leqq t)$ we have $\left[G_{j}: G_{j-1}\right]=2$, we obtain:

$$
\begin{aligned}
2^{t} & =\left[G_{t}: G_{0}\right] \leqq\left[P_{D} N_{E / F}\left(J_{E}\right) J_{F}^{L}: P_{D} N_{E / F}\left(J_{E}\right)\right] \\
& =\left[J_{F}: P_{D} N_{E / F}\left(J_{E}\right)\right] \div\left[J_{F}: P_{D} N_{E / F}\left(J_{E}\right) J_{F}^{L}\right] \\
& \leqq\left[J_{F}: P_{D} N_{E / F}\left(J_{E}\right)\right]=2^{t+1} .
\end{aligned}
$$

This proves the theorem.

Corollary. If $D \neq F^{\times}$(i.e., $V$ is not totally indefinite with respect to $S)$, then it is not possible for $J_{F}^{L} \subseteq P_{D} N_{E / F}\left(J_{E}\right)$. In particular, $J_{F}=P_{F} N_{E / F}\left(J_{E}\right) J_{F}^{L}$, and $\left[J_{F}: P_{D} N_{E / F}\left(J_{E}\right) J_{F}^{L}\right]=2$ if and only if $P_{F} \nsubseteq P_{D} N_{E / F}\left(J_{E}\right) J_{F}^{L}$.

Suppose $D=F^{\times}$. Then, $\left[J_{F}: P_{D} N_{E / F}\left(J_{E}\right) J_{F}^{L}\right]=2$ if and only if 
$J_{F}^{L} \subseteq N_{E / F}\left(J_{E}\right)$ by Lemma. In particular, if $\mathfrak{p}$ is any real spot on $F$, then $\mathfrak{p}$ splits in $E$ and $\delta<0$. Hence, $\delta$ is a totally negative element. Moreover, at each discrete spot we must have $\theta\left(O^{+}\left(L_{\mathfrak{p}}\right)\right) \cong N_{\Re \mid p}\left(E_{\Re}^{\times}\right)$ for $\mathfrak{P} \mid \mathfrak{p}$; equivalently, $\theta\left(O^{+}\left(L_{\mathfrak{p}}\right)\right) \subseteq Q(\langle 1, \delta\rangle)$ over $F_{\mathfrak{p}}$. Note that if $\mathfrak{p}$ does not divide the volume $\operatorname{Vol}(L)$ of $L$, then $L_{\mathfrak{p}}$ is unimodular. Hence, as $r k(L) \geqq 3$ here, $\theta\left(O^{+}\left(L_{\mathfrak{p}}\right)\right)=U_{\mathfrak{p}} F_{p}^{\times 2}$ unless $\mathfrak{p}$ is dyadic and the norm generator has odd order parity with respect to the weight generator for $L_{p}$. See [2]. In the exceptional cases, the spinor norm group is all of $F_{\uparrow}^{\times}$. By the local theory of quadratic forms, we see that $\operatorname{ord}_{\mathrm{p}}(\delta)$ must be even; modulo squares in $F_{p},-\delta$ is a unit of quadratic defect $4 R_{p}$. Hence $E_{\Re} / F_{p}$ must be quadratic unramified. If $\mathfrak{p}$ is an exceptional dyadic prime, then $-\delta \in F_{p}^{\times 2}$. Therefore, the only ramified primes for $E / F$ must also divide $\operatorname{Vol}(L)$. In particular, if $L$ is unimodular, $E / F$ must itself be quadratic unramified. Of course, there are number fields $F$ for which every finite (let alone only quadratic) extension is ramified.

Finally, we point out here that the local lattice representation theory is completely determined when: (i) $\mathfrak{p}$ nondyadic, (ii) $\mathfrak{p}$ unramified dyadic, and (iii) $\mathfrak{p}$ arbitrary dyadic but $L_{\mathfrak{p}}$ modular. For (i) and (ii), see [7]; for (iii), see [8]. Also, see [5] for $\mathfrak{p}$ arbitrary dyadic but $r k\left(L_{p}\right)=2$.

\section{REFERENCES}

1. A. G. Earnest-J. S. Hsia, Spinor norms of local integral rotations, II. Pacific J. Math., 61 (1975).

2. J. S. Hsia, Spinor norms of local integral rotations, I. Pacific J. Math. Vol. 57, No. 1, 199-206 (1975).

3. M. Kneser, Klassenzahlen indefiniter quadratischer Formen in drei oder mehr Veränderlichen, Arch. Math. 7, 323-332 (1956).

4. — Darstellungsmasse indefiniter quadratischer Formen, Math. Zeitschr. 77, 188-194 (1961).

5. O. Körner, Integral representations over local fields and the number of genera of quadratic forms, Acta Arith. 24, 301-311 (1973).

6. O. T. O'Meara, Introduction to Quadratic Forms, Springer-Verlag (1963).

7. The integral representations of quadratic forms over local fields, Amer.

J. Math. 80, 843-878 (1958).

8. C. Riehm, On the integral representations of quadratic forms over local fields, Amer. J. Math. 86, 25-62 (1964).

9. G. L. Watson, Representation of integers by indefinite quadratic forms. Mathematika 2, 32-38 (1955).

10. G. L. Watson, B. W. Jones, On indefinite ternary quadratic forms, Canad. J. Math. 8, 592-608 (1956).

Received October 14, 1975.

The OHio State University 


\section{PACIFIC JOURNAL OF MATHEMATICS}

EDITORS

RICHARD ARENS (Managing Editor)

University of California

Los Angeles, California 90024

R. A. BEAUMONT

University of Washington

Seattle, Washington 98105
J. DugundJI

Department of Mathematics

University of Southern California

Los Angeles, California 90007

D. Gilbarg and J. Milgram

Stanford University

Stanford, California 94305

\section{ASSOCIATE EDITORS}

E. F. BECKENBACH

B. H. NeumanN

F. WOLF

K. YosHIDA

\section{SUPPORTING INSTITUTIONS}

UNIVERSITY OF BRITISH COLUMBIA

UNIVERSITY OF SOUTHERN CALIFORNIA

CALIFORNIA INSTITUTE OF TECHNOLOGY

UNIVERSITY OF CALIFORNIA

STANFORD UNIVERSITY

UNIVERSITY OF TOKYO

MONTANA STATE UNIVERSITY

UNIVERSITY OF UTAH

UNIVERSITY OF NEVADA

WASHINGTON STATE UNIVERSITY

NEW MEXICO STATE UNIVERSITY

UNIVERSITY OF WASHINGTON

OREGON STATE UNIVERSITY

UNIVERSITY OF OREGON

OSAKA UNIVERSITY

AMERICAN MATHEMATICAL SOCIETY
NAVAL WEAPONS CENTER

Printed in Japan by International Academic Printing Co., Ltd., Tokyo, Japan 


\section{Pacific Journal of Mathematics}

\section{Vol. 63, No. $1 \quad$ March, 1976}

Ralph Artino, Gevrey classes and hypoelliptic boundary value problems ....... 1

B. Aupetit, Caractérisation spectrale des algèbres de Banach commutatives .... 23

Leon Bernstein, Fundamental units and cycles in the period of real quadratic

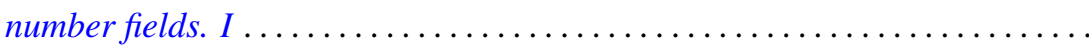

Leon Bernstein, Fundamental units and cycles in the period of real quadratic number fields. II.................................... 63

Robert F. Brown, Fixed points of automorphisms of compact Lie groups ........

Thomas Ashland Chapman, Concordances of noncompact Hilbert cube

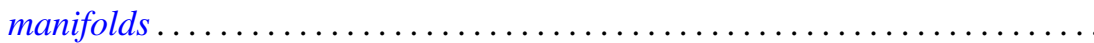

William C. Connett, V and Alan Schwartz, Weak type multipliers for Hankel

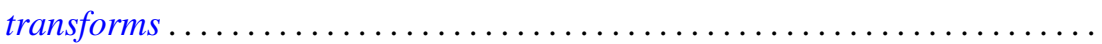

John Wayne Davenport, Multipliers on a Banach algebra with a bounded approximate identity .....................................

Gustave Adam Efroymson, Substitution in Nash functions ................ 137

John Sollion Hsia, Representations by spinor genera ..................

William George Kitto and Daniel Eliot Wulbert, Korovkin approximations in

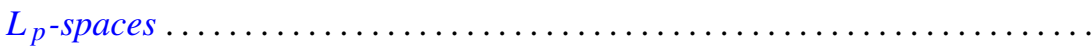

Eric P. Kronstadt, Interpolating sequences for functions satisfying a Lipschitz. condition ...........................................

Gary Douglas Jones and Samuel Murray Rankin, III, Oscillation properties of certain self-adjoint differential equations of the fourth order...

Takaŝi Kusano and Hiroshi Onose, Nonoscillation theorems for differential

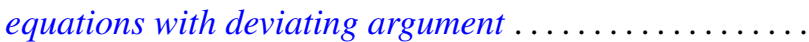

David C. Lantz, Preservation of local properties and chain conditions in commutative group rings. ...

Charles W. Neville, Banach spaces with a restricted Hahn-Banach extension property....

Norman Oler, Spaces of discrete subsets of a locally compact group ...

Robert Olin, Functional relationships between a subnormal operator and its minimal normal extension.

Thomas Thornton Read, Bounds and quantitative comparison theorems for nonoscillatory second order differential equations ...... .

Robert Horace Redfield, Archimedean and basic elements in completely distributive lattice-ordered groups...

Jeffery William Sanders, Weighted Sidon sets

Aaron R. Todd, Continuous linear images of pseudo-complete linear topological spaces.

J. Jerry Uhl, Jr., Norm attaining operators on $L^{1}[0,1]$ and the Radon-Nikodým property.

William Jennings Wickless, Abelian groups in which every endomorphism is a left multiplication. 